Grado en Relaciones Laborales

Facultad de Derecho

Universidad de La Laguna

Curso 2013 / 2014

Convocatoria: Julio

\title{
LA EVALUACIÓN DEL DESEMPEÑO
}

Job performance

Realizado por el alumno Dña. Marta Trujillo Rodríguez.

Tutorizado por la Profesora Dra. Dña. Naira Delgado Rodríguez

Departamento: Psicología Cognitiva, Social y Organizacional

Área de conocimiento: Psicología Social 
Work performance assessment is a structural and systematic process that evaluates the behaviors and results related to the individual performance of the employee in the organization, in order to find out if its productive and it may improve its performance in the future. Performance assessment is very important in order to make decisions with respect to the results that the employees should have, as well as regarding the strategic management of the human capital in the organizations. The present work is divided into three main points. The first point develops the fundamentals that constitute the process of evaluation (concept, characteristics, objectives, interview, implantation, etc). The second point makes reference to the difficulty that exists in the implantation of performance assessment in Public Administrations, despite being collected in the Basic Statute of Public Employee. Last point explains the relationship between performance assessment and motivation.

\begin{tabular}{|l|}
\hline \hline RESUMEN (entre 150 y 350 palabras) \\
\hline La evaluación del desempeño es un proceso estructural y sistemático que \\
permite evaluar los comportamientos y resultados relacionados con el \\
desempeño individual del empleado dentro de la organización, con el fin de \\
conocer si es productivo y si podrá mejorar su rendimiento en el futuro. En \\
la actualidad, la evaluación del desempeño es muy importante para tomar \\
decisiones respecto a los resultados que deben obtener los empleados, así \\
como respecto a la gestión estratégica del capital humano en las \\
organizaciones. El presente trabajo se divide en tres puntos, el primer punto \\
desarrolla los aspectos fundamentales que constituyen el proceso de \\
evaluación (concepto, características, objetivos, entrevista, implantación, \\
etc.). El segundo punto hace referencia a la dificultad que existe en la \\
implantación de la evaluación del desempeño en las Administraciones \\
Públicas, a pesar de estar recogida en el Estatuto Básico del Empleado \\
Público. Por último, la relación que existe entre la evaluación del \\
desempeño y la motivación del empleado. \\
\hline
\end{tabular}




\section{ULL}

\section{Contenido}

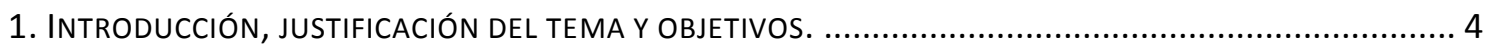

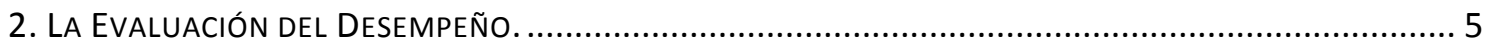

2.1. EVOLUCIÓN DEL CONCEPTO, CARACTERÍSTICAS Y RESPONSABILIDADES.................................... 5

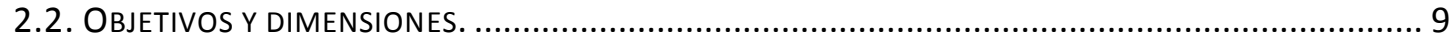

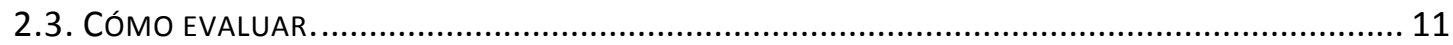

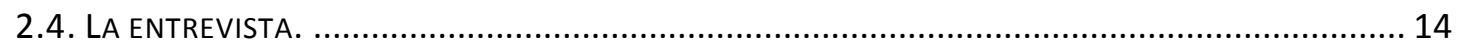

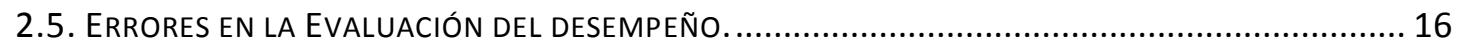

2.6. LA EVALUACIÓN DEL DESEMPEÑO COMO SISTEMA. ......................................................... 18

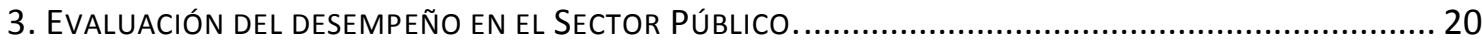

4. ¿EXISTE RELACIÓN ENTRE LA EVALUACIÓN DEL DESEMPEÑO Y LA MEJORA DE LA MOTIVACIÓN DE LOS

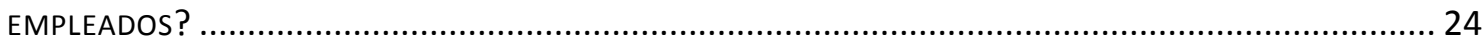

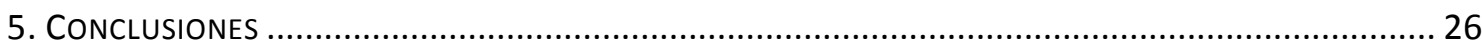

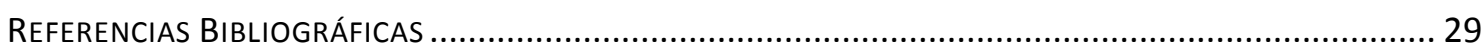




\section{UIII Uninessidad de La Laguna \\ Facultad de Derecho}

\section{INTRODUCCIÓN, JUSTIFICACIÓN DEL TEMA Y OBJETIVOS.}

La evaluación del desempeño es un aspecto básico en la gestión de los Recursos Humanos para la toma de decisiones, ya que con ella se valora de forma objetiva y sistemática el rendimiento que tienen los empleados dentro de la organización. En el presente documento se realiza una descripción de la evaluación del desempeño en las organizaciones, proporcionando la definición de los conceptos básicos que abarca dicha evaluación. El trabajo se divide en tres bloques. El primero de ellos se centra en las características fundamentales que posee la evaluación del rendimiento (dimensiones, métodos, entrevista, errores, implantación, etc.). El segundo bloque indica la importancia que ha adquirido la evaluación del desempeño en la actualidad en las Administraciones Públicas, desde que el Estatuto Básico del Empleado Público la cita como precisa para el óptimo funcionamiento del sector público; concretamente, en este bloque se ha profundizado en las dificultades y los requisitos para que ésta se pueda implantar con efectividad. Por último, se realiza una breve revisión de la relación encontrada en la investigación entre la evaluación de desempeño y la motivación que produce ésta en los empleados. El objetivo del Trabajo Fin de Grado es, por tanto, proporcionar una visión de la evaluación del desempeño amplia y completa, enfocándola no sólo al sector privado sino también al sector público.

Considerando que la evaluación del desempeño ha asumido un papel muy importante en la actualidad debido a la situación de crisis económica por la que pasa el país en los últimos años, la implantación de un sistema de evaluación en una organización no es una tarea sencilla, pero sí necesaria para conocer el verdadero rendimiento y desempeño que tienen los empleados en sus puestos de trabajo. Aunque la productividad es uno de los temas más importantes para las organizaciones, en la actualidad son pocas las empresas que aumentan esta productividad a través del análisis sistemático del rendimiento de sus recursos humanos, debido entre otras cosas al coste económico que supone inicialmente, al estudio previo que deben realizar con la finalidad de establecer objetivos y a los procesos complejos de toma de decisiones que toda implantación de un sistema de evaluación entraña. A lo largo de este trabajo se presentarán los aspectos más importantes a tener en cuenta de cara a valorar la utilidad de evaluar el desempeño laboral de los trabajadores. 


\section{La EvaluaCión DEL Desempeño.}

\subsection{EVOLUCIÓN DEL CONCEPTO, CARACTERÍSTICAS Y RESPONSABILIDADES.}

El concepto "evaluación del desempeño" nace en la Edad Media con la Compañía de Jesús, fundada por Ignacio de Loyola, la pionera en la realización de un sistema combinado de informes y de calificaciones que los miembros de la orden preparaban de sí mismos. Alrededor de 1842, el servicio público de EEUU implantó un sistema de informes anuales para evaluar el desempeño de sus trabajadores, pero no fue hasta después de la Segunda Guerra Mundial cuando estos sistemas comenzaron a difundirse en las organizaciones.

El desempeño ha sido definido a lo largo de la historia por diversos investigadores. Por ejemplo, la Society for Industrial and Organizational Psychology (1987) definía al desempeño como la efectividad y el valor de la conducta laboral y sus resultados, es decir, que el desempeño era igual a la efectividad o la productividad, por lo que no existía una definición específica. Fue Murphy (1990) quien separó estos tres términos, definiendo la productividad como el resultado entre las variables resultantes y las variables invertidas, éste afirmaba que el tiempo de trabajo no está dedicado sólo a realizar tareas identificables en un análisis del puesto. Por lo que el desempeño es el conjunto de conductas que son relevantes para las metas de la organización o la unidad organizativa en la que la persona trabaja. Murphy diferencia cuatro tipos de conductas generales en los puestos de trabajo, estas son: conductas orientadas a la tarea, las que están orientadas interpersonalmente, las que están relacionadas con la pérdida de tiempo y las conductas destructivas.

La concepción explicada en el párrafo anterior fue ampliada y desarrollada por otros investigadores. John P. Campbell y sus colegas en 1990 (citado en Salgado y Cabal, 2011) definieron el desempeño como cualquier conducta cognitiva, psicomotora, motora o interpersonal, bajo el control del individuo, graduable en términos de habilidad y relevante para las metas organizacionales.

En esta línea, cuando hacemos referencia a la evaluación del desempeño desde la óptica de la Gestión de los Recursos Humanos, el desempeño queda definido como el 


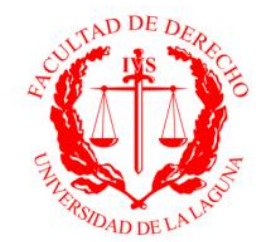

proceso por el cual se identifican, observan, miden y valoran los resultados y comportamientos que están bajo el control del evaluado y que resultan relevantes para una organización determinada (Carroll y Schneier, 1982). Esta definición alude a un proceso amplio, en el que la identificación previa de los factores críticos de rendimiento, la medición de dichos factores y la recogida de información son tan valiosas como el propio momento de las decisiones de evaluación.

En cuanto a las características del concepto debemos tener claro el significado de rendimiento y desempeño, ya que se utilizan habitualmente como sinónimos y no lo son. Los términos "rendimiento" o desempeño" comparten connotaciones de producto, resultado o ejecución, pero la definición de éstos no es la misma. Cuando hablamos de rendimiento lo relacionamos en mayor medida con indicadores corporativos, financieros o de negocio, en cambio cuando hacemos referencia al término desempeño estamos estableciendo una relación directa con la persona y su compromiso de realización de una determinada labor. Dicho esto, podemos hablar entonces de diferentes niveles de desempeño o rendimiento dentro de la organización.

Antes de que podamos evaluar el desempeño en un empleado debemos conocer las tareas y las responsabilidades que el individuo lleva a cabo, así como el criterio de un desempeño dinámico y saber si existen actividades más relevantes que otras.

El desempeño individual se evalúa en función de los niveles superiores, por eso debe contemplar hasta qué punto todas las dimensiones de orden superior, desde la demostración de los valores corporativos hasta la superación de los objetivos cuantitativos, se reflejan en el comportamiento profesional de los empleados. Este tipo de desempeño (individual) está sometido a todo un conjunto de circunstancias externas que condicionan el resultado final, y cuya consideración es imprescindible para que su evaluación sea considerada adecuada por parte de los empleados.

La evaluación del desempeño es una apreciación sistemática de cómo cada persona se desenvuelve en un puesto y de su potencial de desarrollo futuro. Existen varios procedimientos de evaluación individual en una organización, éstos pueden ser evaluación de méritos, evaluación de la eficiencia en las funciones, informes de avance, 
etc, siento la evaluación de desempeño una técnica de administración imprescindible dentro de la actividad administrativa.

Además de las características señaladas, se pueden diferenciar dos tipos de evaluación: la informal y la formal. Jones y George (2006) definen la evaluación informal como un estudio no programado, conociendo los avances y los aspectos que se deben reforzar a través de la retroalimentación, por medio de entrevistas, observaciones, revisión de documentos y archivos de los trabajadores (Urdaneta y Urdaneta, 2013)

Por otro lado, la evaluación formal es un proceso que se da de manera programada, cada año, a diferencia de la informal que es continua y no programada. Jones y George (2006:436) la definen como una evaluación “'llevada a cabo en fecha fija durante el año y con base en las dimensiones y medidas de desempeño que se especificaron por adelantado". De esta manera, las evaluaciones formales permiten que el empleado conozca su resultado respecto al desempeño actual, y permite la comparación de desempeño de distintos empleados, identificando a aquellos empleados que merecen aumentos por méritos y a los que necesitan mayor capacitación (Urdaneta y Urdaneta, 2013)

Para que la evaluación del desempeño se emplee adecuadamente en la organización, la dirección de RRHH debe implantar los siguientes pasos:

- Garantizar que exista un clima laboral de respeto y confianza entre las personas,

- Propiciar que las personas asuman responsabilidades y definen metas de trabajo.

- Desarrollar un estilo de administración democrático, participativo y consultivo.

- Crear un propósito de dirección, futuro y mejora continua de las personas.

- Generar una expectativa permanente de aprendizaje, innovación, desarrollo personal y profesional.

- Transformar la evaluación del desempeño en un proceso de diagnóstico de oportunidades de crecimiento, en lugar de que sea un sistema arbitrario, basado en juicios.

Como se señala, estos pasos serán fundamentales para una eficaz implantación del sistema de evaluación en la empresa, y será la dirección de Recursos Humanos la 

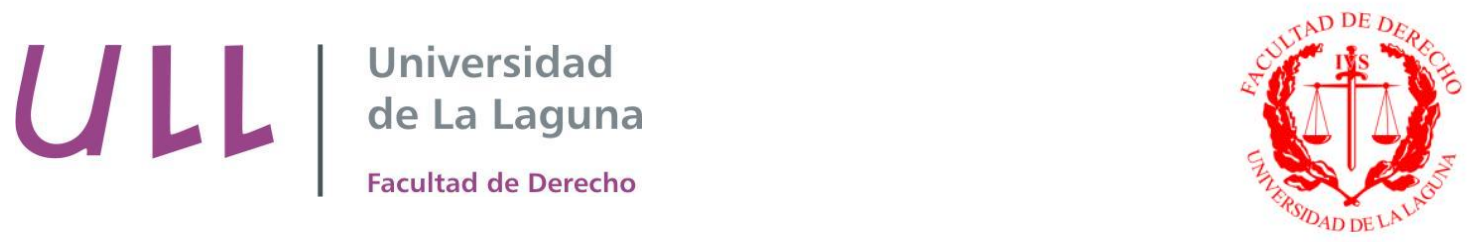

encargada de llevar a cabo cada uno de los puntos señalados anteriormente y así conducir a la organización al éxito.

La atribución de la responsabilidad de la evaluación del desempeño es múltiple, dependiendo de la política de recursos humanos que posea cada empresa. A continuación se hace referencia a las personas que tienen ese grado de responsabilidad para que la implantación sea efectiva (Chiavenato, 2007):

- El gerente: Asume la responsabilidad del desempeño de sus subordinados y de su evaluación, debido a la falta de conocimientos para desarrollar el plan de evaluación le asesora el área encargada de la administración de personas.

- El propio empleado: Esta responsabilidad se da en organizaciones que tienen como características el ser democráticas y modernas, siendo el propio empleado quien se autoevalúa en su propio puesto.

- El empleado y el gerente: Actualmente la administración por objetivos (APO) tiene como fundamentos el ser democrática, motivadora y participativa entre otras. Orientando la evaluación de esta manera: formulación de objeticos mediante consenso, compromiso personal para alcanzar los objetivos formulados conjuntamente, acuerdo y negociación con el gerente respecto a la asignación de los recursos y a los medios necesarios para alcanzar los objetivos, desempeño, medición constante de los resultados y comparación con los objetivos formulados, retroalimentación intensiva y continua evaluación conjunta.

- La comisión de evaluación: Evaluación colectiva que está realizada por un grupo de personas, formada por personal que pertenece a diferentes departamentos o áreas. Es criticada por su centralización y por su enjuiciamiento.

- Evaluación de $360^{\circ}$ : Hace referencia al contexto general que envuelve a cada persona, de forma circular, se evalúa por todos los elementos que tienen interacción con el individuo evaluado, participando en ella, el superior, los colegas, los subordinados, los clientes, los proveedores, etc. Una de las ventajas es que es un sistema amplio y que las evaluaciones provienen de muchas perspectivas, pero a la misma vez es complejo porque dichas evaluaciones deben ser combinadas. 


\section{ULL}

\subsection{OBJETIVOS Y DIMENSIONES.}

Cuando en una organización se realizan sistemas de evaluación de desempeño se busca un objetivo general, éste puede ser el de optimizar los resultados de la organización modulando los comportamientos del empleado para optimizar o maximizar el rendimiento corporativo. Cuando la finalidad de los sistemas de evaluación es el control, hacemos referencia a las políticas de compensación (comisiones, bonus anuales, etc.). Pero éste no es el único objetivo que puede perseguir la evaluación de desempeño, ya que fomentar el desarrollo profesional de los empleados es otro objetivo general, en este caso se busca dar información y retroalimentación a los empleados sobre su desempeño para que de esta manera puedan evolucionar en su rol profesional y su carrera.

Además de los dos objetivos generales señalados, la evaluación de desempeño reúne otras funciones muy importantes dentro de la organización. En primer lugar, la comunicación interna de las variables críticas de rendimiento porque los formularios por los que serán evaluados son un instrumento de comunicación. En segundo lugar, la validación de otras políticas de Recursos Humanos dentro de la organización, esto es así porque la información es una herramienta fundamental para la adecuación de otras áreas de Recursos Humanos. En tercer lugar, la identificación y gestión de talento, ya que aporta información sobre el potencial del empleado para futuros puestos (Ángel, Dubin, Salvador y Simón, 2008).

El sistema de evaluación de desempeño tiene un carácter multifuncional, por esta razón la organización debe definir el fin o los objetivos que desean con la implantación del sistema, para que de esta manera el impacto sea positivo. En este sentido, Gorriti en su artículo indica que los propósitos para los que se puede hacer una Evaluación del desempeño son los siguientes (Cleveland, Murphy y Williams, 1989):

- Evaluación entre personas: para el aumento de salarios a través de incentivos y la promoción, aportando de esta manera una oportunidad de ascenso al empleado.

- Evaluación de personal: identificar los fallos o errores del empleado para poder modificarlos y conocer cuáles son sus puntos fuertes en el desempeño de la tarea. 


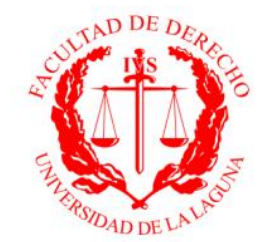

- Mantenimiento del sistema: Investigar para adquirir conocimiento que será utilizado para la gestión de los Recursos Humanos y para una efectiva planificación en la organización.

- Documental: para justificar decisiones e intervenciones ante recursos o cualquier otro proceso administrativo o legal implicado.

Respecto a las dimensiones que recoge una evaluación de desempeño, actualmente se diferencian tres ámbitos de desempeño para la organización: el desempeño referido a tarea, el desempeño contextual y las conductas contraproductivas. La primera hace referencia a actuaciones directamente exigidas para la ejecución de las tareas, se trata de los comportamientos que crean el producto o servicio que satisface las responsabilidades que asume la organización. El principal predictor de la ejecución de tarea es la aptitud cognitiva (Schmidt y Hunter, 1998; Ones, Viswesvaran y Dilchert, 2005) formada por los conocimientos del puesto, las destrezas entendidas como formas estandarizadas de resolver problemas (Campbell y Kuncel, 2001) y los hábitos entendidos como respuestas características (Borman, Penner, Allen y Motowidlo, 2001). En segundo lugar, se encuentra el desempeño contextual, que se refiere a un comportamiento de naturaleza social, que actúa como catalizador del desempeño de tarea facilitándolo o dificultándolo. Para Borman y Motowidlo (1993) las razones por las que es importante evaluar el desempeño contextual son porque definen el ambiente de trabajo, son comunes a la mayoría de los trabajos de una organización, son más motivadoras que cognitivas y porque aunque deseables, son más implícitas que explicativas (Gorriti, 2007). Algunos ejemplos de conductas que definan el desempeño contextual son: Aportar ayuda a compañeros de trabajo ofreciéndoles sugerencias, ,mostrar consideración, cortesía y tacto en las relaciones así como demostrar motivación y confianza, representar a la organización eficazmente defendiéndola y expresando satisfacción y lealtad más allá de momentos o circunstancias. Por último, otra dimensión del desempeño laboral son las conductas contraproductivas, la definición más utilizada de las mismas es la propuesta por Sackett y DeVore (2001), que señala que se trata de "toda conducta intencional por parte de un miembro de la organización vista por ésta como contraria a sus legítimos intereses". Citar los siguientes ejemplos de conducta contraproductivas: destrucción de propiedades de la organización, mal uso del 

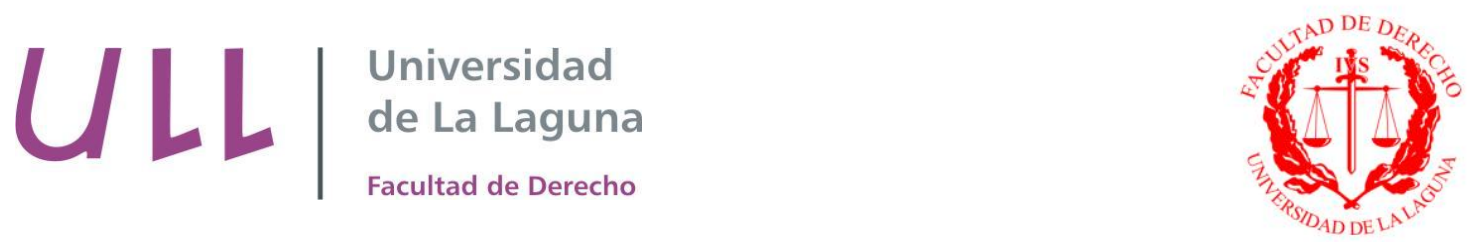

tiempo y de los recursos, abusos físicos o sexuales, uso de correo electrónico para fines personales, hacer negocios personales en tiempo de trabajo, uso y abuso de alcohol, etc.

\subsection{Cómo EVALUAR.}

Para que un sistema de evaluación del rendimiento sea válido deben especificarse los criterios de rendimiento que resulten importantes, debiendo de estar relacionados con el puesto de trabajo y ser de fácil identificación. A través del análisis de puestos de trabajo se identifican varios de estos criterios que reflejan las contribuciones del empleado (desempeño de tarea).

Cuando el sistema de evaluación no aborda el comportamiento en el trabajo y los resultados que se hayan determinado en el análisis del puesto, se dice que es un sistema deficiente. Cuando el sistema incluye aspectos que no son relevantes para el trabajo, se dice que éste estará contaminado. Si un puesto abarca varios criterios es necesario realizar una ponderación de los mismos, de esta manera se reconocerá en la evaluación la importancia que tiene cada uno de ellos en la consecución de los objetivos.

Uno de los aspectos que puede requerir ponderación es el hecho de que se tenga en cuenta la evaluación que realizan diferentes agentes de un mismo empleado. En este sentido, la evaluación puede llevarse a cabo por múltiples evaluadores.

En primer lugar la evaluación por parte de los superiores, siendo el superior el jefe inmediato del subordinado al que se evalúa. Éste es una de las principales fuentes de información, ya que, debe conocer el puesto de trabajo y su rendimiento. Algunos de los inconvenientes de que sea el superior quien realice la evaluación son: la amenaza que supone para los trabajadores, dado su poder de recompensar y sancionar; que se produzca una actitud defensiva del subordinado por la falta de diálogo; que el jefe no proporcione retroalimentación por la falta de habilidades interpersonales.

En segundo lugar la autoevaluación, esto es cuando los empleaos participan en la evaluación de su propio desempeño o rendimiento. Este uso incide positivamente en el grado de compromiso que adquiere el individuo con los objetivos, siendo una herramienta efectiva en los programas que se centran en el desarrollo y crecimiento 
personal, y en el compromiso con los objetivos. A veces, este sistema se tiene en cuenta conjuntamente con la evaluación del superior, realizando una comparación entre ambas.

En tercer lugar la evaluación por partes de los iguales, cuando los superiores no tienen información sobre alguno de los comportamientos de los subordinados se acude a los iguales, pero la competitividad provoca un bajo grado de confianza entre los subordinados.

En cuarto y quinto lugar se encuentra la evaluación por parte de los subordinados y la evaluación por parte de los clientes del titular puesto. Cuando son los subordinados los que ejecutan la evaluación se puede lograr que los superiores sean más conscientes de los efectos que éstos provocan y de los fallos que pueden tener como tales. La evaluación por parte de los clientes puede resultar adecuada en diversos contextos, ya que conocer cuáles son los puntos de satisfacción e insatisfacción es muy importante de cara a corregir o estimular comportamientos.

Por último, añadir la evaluación 360 grados como el método que compendia todos los anteriores y que tiene como objetivo disponer de más de una fuente o perspectiva en el sistema de evaluación. Otra tendencia a destacar es el seguimiento informático que consiste en la recogida de datos mediante sistemas informáticos, respetando siempre la intimidad de los empleados.

El momento y el contexto de la evaluación es un aspecto muy importante a la hora de realizar la valoración, ya que, si ésta no se hace en el momento adecuado, puede transmitir una imagen falsa de la persona, generando frustración e insatisfacción en el evaluado.

Los métodos de evaluación varían de una organización a otra, ya que cada una de ellas construye su propio sistema específico que dependerá del nivel y de las áreas de adscripción del personal, cada uno de éstos se sujeta a unos objetivos determinados y a las características del personal implicado. No debemos olvidar que la evaluación del desempeño es un medio para obtener información y datos que se procesarán para mejorar el desempeño humano en las organizaciones, es un método y una herramienta, pero no es un fin en sí misma.

A continuación se hará mención de los principales métodos de evaluación del desempeño y se explicará brevemente cada uno de ellos: 
- Método de evaluación del desempeño mediante escalas gráficas.

- Método de elección forzosa.

- Método de evaluación del desempeño mediante incidentes críticos.

- Método de comparación por pares.

El método de evaluación del desempeño mediante escalas gráficas es el más empleado y el más simple, permite medir el desempeño de las personas empleando factores previamente definidos y graduados, utilizando un cuestionario de doble entrada en el cual se representarán los factores en las líneas horizontales y los grados de variación en las líneas verticales. Una de las ventajas más relevantes de este método es que permite una visión integral y resumida de los factores de evaluación, es decir, refleja las características del desempeño más importantes para la empresa. Respecto a las desventajas que supone la utilización de este método, podemos señalar que no brinda flexibilidad al evaluador, que tiende a caer en rutina y a estandarizar las respuestas. Este método se debe emplear con máximo cuidado para evitar desviaciones, un ejemplo es el "Criterio nebuloso" en el que es necesario emplear frases descriptivas que definan con precisión cada factor evaluado, ya que, de lo contrario, los ítems se pueden interpretar de diferente manera y eso produciría confusión y falta de equidad.

El método de elección forzosa consiste en la evaluación del desempeño de los empleados mediante frases descriptivas de determinadas alternativas de tipos de desempeño individual. Hay dos formas de composición:

- Se forman bloques de dos frases de significado diferente, generalmente dos de significado positivo y dos de significado negativo, para valorar al empleado. De cada bloque, el supervisor o evaluador elige la frase que más se ajusta, y luego, la que menos se ajusta al desempeño del evaluado.

- Se forman bloques de sólo cuatro fases de significado positivo, y el evaluador elige las frases que más se ajustan al desempeño del evaluado.

Las frases que conforman los conjuntos o bloques no se escogen al azar, sino que deben seleccionarse de manera razonable mediante un procedimiento estadístico tendente a verificar su adecuación a los criterios existentes en la empresa y su capacidad de discriminación, a través de dos índices: el de aplicabilidad y el de discriminación. Su 


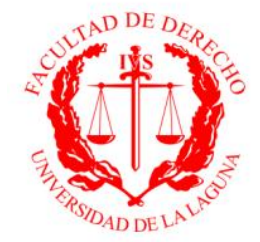

principal ventaja es que evita distorsiones introducidas por los evaluadores, tales como el sesgo de benevolencia o el efecto halo.

El método de evaluación del desempeño mediante incidentes críticos fue desarrollado en la Segunda Guerra Mundial por Estados Unidos, basándose en que el comportamiento humano posee características externas que pueden ser positivas, lo que conocemos con el éxito, o negativas (fracasos). En torno a las situaciones excepcionales positivas y negativas de desempeño de las personas se construye el instrumento de evaluación.

El método de comparación por pares es un proceso simple y en general poco eficiente, consiste en comparar a los empleados de dos y en dos, apuntando lo que se considera mejor respecto al desempeño a la derecha. Este método es aplicable cuando los evaluadores no tienen medios suficientes para emplear otros métodos que son más eficientes y completos.

Añadir que la evaluación de desempeño se ha visto repercutida por la era de la información, aportando de esta manera: dinamismo, cambio y competitividad. En la actualidad, prevalece el esfuerzo por una evaluación cualitativa de las personas que se realiza directamente y que no depende de informes continuos, buscando la excelencia pro medio de inversiones en educación, capacitación y desarrollo de las personas.

Para finalizar, señalar dos factores muy importantes que han marcado el curso de la evaluación del desempeño. El primero es la sustitución de una organización dividida en departamentos por una formada por equipos, alterando así los sistemas de indicadores y de mediciones dentro de la empresa. En segundo lugar, los trabajadores participan en los resultados de la empresa requiriendo un sistema de mediciones y de indicadores que permita negociar entre ellos y sus administradores.

\subsection{LA ENTREVISTA.}

Es una herramienta muy importante en el sistema de evaluación del desempeño, debe reunir información válida y necesaria o de lo contrario no es efectiva, por lo que hay que tener en consideración varios aspectos. Se debe obtener información pertinente, actualizando expedientes y efectuando autoevaluaciones, comparando ésta con la 


\section{UII $\left.\right|_{\text {ninessitad }} ^{\text {U. }}$

apreciación del supervisor y pudiendo utilizarse en la entrevista como un tema de debate.

Existen varios tipos de entrevista, pero a continuación citaremos cuatro de las más importantes (Dolan, Cabrera, Jackson y Schuler, 2007):

1. Hablar y convencer: Se conoce con el nombre de entrevista directiva, se transmite al empleado cómo lo está haciendo y se le inclina a instaurar objetivos concretos para su mejora en el desempeño.

2. Hablar y escuchar: Se caracteriza porque los sujetos evaluados posean habilidades de pregunta y escucha, dando la posibilidad de participar y de poder crear un diálogo con sus superiores, dejando en evidencia los puntos fuertes y débiles de los trabajadores. Este tipo de entrevista no sigue ningún formato rígido.

3. Solución de problemas: En primer lugar se debe establecer un diálogo abierto y activo, se comparten impresiones y se buscan soluciones a los problemas que los subordinados puedan presentar. Este tipo de entrevista se caracteriza por la dificultad que tienen algunos superiores a la hora de debatir abiertamente aspectos de la evaluación con los empleados, requiriendo que los evaluadores posean una formación específica.

4. Entrevista mixta: Es un mezcla de la entrevista de "hablar y convencer" y de la de "solución de problemas", los evaluadores deben estar formados y poseer las suficientes habilidades para llevar a cabo los diversos fines de la entrevista. Puede empezar con el trabajador que será evaluado que escuchará lo que el superior le expone sobre su desempeño, seguido de un debate participativo para soluciones los posibles problemas y estableciendo objetivos de mejora. La retroalimentación debe ser efectiva, ya sea positiva o negativa.

La comunicación del resultado de la evaluación es un punto esencial en un sistema tan relevante como la evaluación del desempeño, proporcionando la información relevante y significativa. De esta manera, la entrevista sirve para establecer la comunicación entre superiores y subordinados. Los propósitos de la entrevista pueden ser (Chiavenato, 2007):

- Manifestar al evaluado las condiciones que debe mejorar en su trabajo de forma clara cuál es el modelo de desempeño que debe seguir.

- El empleado debe tener una idea de cómo está desempeñando sus tareas, es lo que se conoce como retroalimentación, siendo necesario que éste sepa lo que su 


\section{ULL}

superior opina de su trabajo y así poder cambiar y ajustar su desempeño al esperado por sus superiores.

- Discutir las diversas medidas y planes que orienten al evaluado a mejorar las aptitudes y a participar activamente.

- Crear un ambiente en el que la relación entre el evaluador y los evaluadores sea fuerte y sólida, logrando así que el subordinado se exprese francamente y desaparezca la tensión que se pueda crear cuando no se recibe una asesoría planeada y orientada.

El éxito depende de muchos factores y debe ser preparada de modo que el evaluador sepa de antemano lo que dirá el evaluado y cómo lo hará, teniendo claro que todo trabajador tiene aspiraciones y objetivos particulares.

Steensma y Otto (2000) concluyen lo siguiente:

- Cuanto mayor es el liderazgo participativo durante las sesiones de evaluación, mayor es la satisfacción del empleado.

- Cuanto mejores son las técnicas de comunicación durante las sesiones de evaluación, mayor será la satisfacción del evaluado.

- Cuanto mejor es la información del proceso de evaluación que se da de antemano a los empleados, mayor satisfacción se logra.

En definitiva, la entrevista es un aspecto crítico del sistema, ya que el evaluado valora de forma positiva o negativa dicho sistema a través de los elementos que percibe, esto se conoce como justicia del procedimiento y tiene que ver con las diferentes percepciones entre supervisores y evaluados, así como la confianza en los sistemas internos y la relación que la organización crea con el empleado evaluado.

\subsection{ERRORES EN LA EVALUACIÓN DEL DESEMPEÑO.}

La evaluación del desempeño presenta como principal problema el almacenamiento de la información por largos períodos de tiempo, pudiendo ser recuperada cuando fuera necesario o para emitir los informes requeridos. Señalar que existe una pluralidad de actitudes, estereotipos y creencias por parte de los evaluadores a la hora de realizar la evaluación. 
En ocasiones, los evaluadores deben decidir qué información se le facilitará a los empleados, y esta decisión se realiza conociendo las posibles consecuencias que acarreará, ya que el empleado podría quedar descontento y sus oportunidades de ascenso se podrían ver limitadas.

En relación con el superior, que es generalmente el evaluador, se plantean varios problemas como el desconocimiento del puesto de trabajo y de las tareas que el evaluado realiza habitualmente, la evaluación indebida por la inestabilidad que provocan las medidas y las calificaciones, y también por la utilización de medidas inadecuadas.

Cuando no se definen los criterios con la claridad que se precisa se pueden producir una serie de errores de apreciación durante la evaluación de desempeño, éstos son :

- Efecto halo y eco: El efecto halo consiste en que el individuo será evaluado de manera similar en todas aquellas dimensiones de rendimiento que posea éste, basándose en la dimensión que percibe y que considera más relevante. El efecto eco es lo opuesto al efecto halo, éste se da cuando una pésima calificación influye o afecta al resto de la evaluación.

- Error de lenidad y error de severidad: El error de lenidad también conocido como el de indulgencia consiste en que el evaluador califica por encima de la calificación que realmente debería tener el empleado evaluado. En el caso opuesto destacar el error de severidad, los individuos que están sometidos a la evaluación son calificados por debajo de su nivel de rendimiento.

- Error de tendencia central: El evaluador da valores a los sujetos que se encuentran en el medio de la escala, siendo consecuencia de los métodos de evaluación.

- Efectos de contraste: Este efecto surge por la comparación que el evaluador realiza entre los evaluados, quedando afectada la evaluación del rendimiento de forma indebida.

- Error de semejanza: Este error se da cuando el evaluador se ve reflejado en alguno o algunos de los evaluados, dando una calificación más favorable o positiva a éstos.

La necesidad de fijar cuáles son los fines a los que quiere llegar la organización mediante la evaluación de desempeño es fundamental para evitar o disminuir errores posteriores. Teniendo en cuenta una clase de criterios, como por ejemplo la economía 
(coste de elaboración), facilidad a la hora de implantar el método, que sea aceptable por parte del usuario, etc.

Las diferencias entre el actor (evaluado) y el observador (evaluador) son uno de los principales problemas a la hora de realizar la evaluación, ya que cada uno ve el proceso desde un punto de vista diferente. Centrándose de esta manera el evaluador en la motivación y en las diversas habilidades que pueda mostrar el individuo, en cambio el evaluado se basa en factores externos. Estas diferencias ocasionan una gran problemática a la hora de buscar las posibles causas de un rendimiento deficiente, ya que, existe la predisposición de manifestar los resultados del rendimiento en beneficio propio.

\subsection{LA EVALUACIÓN DEL DESEMPEÑO COMO SISTEMA.}

Cuando se hace mención al Sistema de Evaluación se habla de innovación organizacional, por lo que se debe conocer el estado de la empresa y el objetivo que se desea conseguir, teniendo en cuenta la cultura empresarial y los valores de la organización.

La comunicación de la implantación y sus objetivos adquiere un gran papel, ya que cuando ésta mal gestionada provoca en el personal una gran confusión, una negatividad hacia el sistema, la motivación disminuye, etc. Debe ser la alta dirección quien asuma la responsabilidad de que exista una efectiva gestión, divulgando la importancia que corresponde y dedicando el tiempo y los recursos que exija la implantación. Por otra parte, para que la aplicación del sistema sea coherente la alta dirección será evaluada puntualmente con la finalidad de manifestar la seriedad y la verdadera aplicabilidad.

La correcta formación de los evaluadores es fundamental para evitar los posibles errores nombrados en el anterior punto, ya que éstos deben reunir la formación necesaria para evaluar a través del método elegido de una forma efectiva. Se debe tener en cuenta la motivación, tanto en los evaluadores como en lo evaluados, siendo ésta esencial para llevar a cabo la evaluación. 


\section{ULL}

La revisión o la evaluación constante del propio sistema que se quiere implantar es necesaria para que no exista deficiencia, de esta manera se recomienda crear un comité que se encargará de cuidar la calidad y la eficacia del sistema. Destacar algunas de las funciones que posee dicho comité :

- Estimar la estabilidad y la fiabilidad de las medidas tomadas, y que éstas sean válidas.

- Que exista igualdad entre las unidades a la hora de efectuar el proceso.

- La aplicación debe ser justa.

- Analizar si el sistema está ligado a la cultura y a los objetivos de la organización.

Si existiera algún cambio en el sistema tras su estudio, este cambio deberá ser comunicado a los empleados y a los directivos en líneas, ya que la comunicación es principal para asumir los cambios y corregir los posibles errores.

Uno de los principales fundamentes en la evaluación del desempeño es la toma de decisiones respecto a los resultados que han obtenido los empleados, estas decisiones pueden ser favorables o negativas cuando el rendimiento no es el esperado. Algunas de las decisiones más comunes son el despido, aumento o reducción salarial, promoción, etc. No sólo es importante el hecho de tomar este tipo de decisiones, sino que también deben facilitar que el empleado perfeccione el desarrollo de su puesto de trabajo, por ejemplo a través de la motivación y la retroalimentación. Una técnica cada vez más utilizada es la autogestión de los propios empleados, que consiste en la identificación de sus propios problemas y establecer sus soluciones, así como sus propias metas.

La valoración del sistema de evaluación es necesaria si efectivamente se han logrado los objetivos que se habían implantado desde un principio, revisando los métodos que se han empleado y si fuera necesario mejorarlo para su óptima aplicación y así lograr la efectividad.

Por lo tanto la evaluación del desempeño y su valoración son esenciales para que la organización pueda tomar las decisiones relacionadas con la Gestión de los Recursos Humanos, obteniendo una base sólida de información sobre el rendimiento, las mejoras y las deficiencias que presentan los empleados dentro de la empresa. La gestión del desempeño debe estar integrada en todas las políticas de Recursos Humanos para que se logre la realidad deseada. 


\section{UII $\left.\right|_{\text {nniversidad }} ^{\text {Und }}$ de La Laguna \\ Facultad de Derecho}

\section{Evaluación del Desempeño en el Sector Público.}

Implantar la evaluación del desempeño en las Administraciones Públicas es un reto fundamental desde que el Estatuto Básico del Empleado Público (en adelante, EBEP) la ubica como una obligación legal. Dicho estatuto establece en su artículo 20.1 que el desempeño es el procedimiento mediante el cual se mide y valora la conducta profesional y rendimiento o logro de resultados, en el artículo 20.3 los efectos serán en la carrera profesional horizontal, la formación, la provisión de puestos de trabajo y en la percepción de las retribuciones complementarias. Además, dice en su artículo 20.4 que también tendrá efecto en la continuidad de un puesto de trabajo obtenido por concurso. De esta manera, la evaluación del desempeño se plantea como un mecanismo esencial, adaptando su planteamiento y contenido a los principios de igualdad, objetividad y transparencia, pero implantando dicha herramienta para la carrera profesional, el sostenimiento de los puestos de trabajo y la determinación de las retribuciones complementarias.

Gorriti (2007) expone en su artículo que el problema de que la implantación de este procedimiento sea mínimo es la necesidad de hacerlo de forma sistemática y con un método contrastado. Éste indica que la necesidad de la evaluación del desempeño no está en cuestión, de hecho es inevitable, lo que se ve necesario es incorporarla como una práctica cotidiana y profesional de las actividades naturales de los Recursos Humanos en las Administraciones Públicas. Cuando una Administración no evalúa su desempeño, obstaculiza el cambio gradual y está condenada a cambios cualitativos y traumáticos originados por una modificación política, tecnológica, etc.

Salgado y Cabal (2011) clarifican el comportamiento que deben tener los empleados públicos, para ello se acude al artículo 52 del EBEP, que indica que dichos empleados deberán desempeñar con diligencia las tareas que tengan asignadas, por lo que el desempeño de tarea debe ser evaluado. También se hace referencia al artículo 53 y 54, que reflejan la conducta y los principios éticos relevantes de cara al desempeño, a continuación señalamos algunos de los apartados de estos artículos del EBEP:

- Los empleados públicos deben perseguir la satisfacción de los intereses generales de los ciudadanos, estando ajustada a los principios de lealtad, buena fe, eficacia, economía y eficiencia, vigilando de esta manera el cumplimiento de los objetivos de la 


\section{UIII Uninesisiad

organización. Cumpliendo con diligencia las tareas que les corresponda o se les encomienden dentro del plazo (artículo 53 EBEP).

- Tratarán con respeto a sus superiores, a los ciudadanos y todo el conjunto de empleados públicos. En cuanto al desempeño de las tareas deberá ser correspondiente a su puesto de trabajo, que se realizará de forma diligente, cumpliendo la jornada y los horarios. Los empleados tendrán que mantener su formación y cualificación actualizada, pudiendo poner en conocimiento a sus superiores o a los órganos competentes las propuestas que consideren de mejora (artículo 54 EBEP).

Además de los artículos señalados, también están los que reflejan las conductas contraproductivas que se consideran faltas muy graves :

- "El abandono del servicio, así como no hacerse cargo voluntariamente de las tareas o funciones que tienen encomendadas" (Art. 95.2c),

- "El notorio incumplimiento de las funciones esenciales inherentes al puesto de trabajo o funciones encomendadas" (Art. 95.2g),

- "La desobediencia abierta a las órdenes o instrucciones de un superior" (Art. 95.2i).

Por lo que el Estatuto Básico del Empleado Público instaura tres tipos de conductas laborales que se corresponden con las dimensiones de desempeño nombradas anteriormente (desempeño de tarea, desempeño contextual y conductas contraproductivas).

Uno de los requisitos es el conjunto de "reglas del juego" vigente en el ámbito de gestión de recursos humanos de las Administraciones Públicas, estas "reglas del juego" a nivel informal, generan una regulación a partir de los principios genéricos establecidos en la normativa, desarrollando una interpretación e incidiendo sobre el comportamiento de los agentes involucrados. Varios estudios han señalado algunos de los aspectos que caracterizan el ámbito informal, asociándolos a los valores que caracterizan el sistema de gestión de los recursos humanos. Entre éstos cabe destacar:

- La idea de igualdad, aplicada en el diseño e interpretación de los mecanismos de acceso al puesto de trabajo, de acceso a la carrera administrativa o en la configuración del sistema salarial y de incentivos.

- El mérito y la capacidad como principios que regulan el acceso y el desarrollo de la carrera profesional, aunque básicamente medidos a partir de su cumplimentación formal. 
- La unidad, asociada a la uniformidad en la configuración del sistema de función pública que se extiende a los empleados públicos con vínculos laborales no estatutarios.

- Centralización de la gestión y administración de las principales actividades vinculadas a la gestión de personal en el interior de las Administraciones Públicas.

- La jerarquía como principal mecanismo de coordinación entre unidades.

- La seguridad de las condiciones de los empleados públicos, planteada como garantía frente a posibles arbitrariedades de la dirección política.

Esto no resulta de fácil aplicación en el contexto institucional, por lo que una labor previa debería consistir en el reconocimiento de estas “ reglas del juego” y en el proyecto de una estrategia de cambio institucional conforme con el nuevo enfoque que se desea introducir (Ángel, Dubin, Salvador y Simón, 2008).

Existen una series de dificultades de carácter operativo, cultural y de resistencia al cambio por parte de diferentes colectivos, que han paralizado la prolongación de esta herramienta, como puede ser la carencia de cultura de evaluación, la proximidad entre evaluadores y evaluados, y la ausencia de incentivos para desarrollar roles directivos y de gestión de personal en el nivel de dirección de línea que acaban desvirtuando el sistema, flexibilizándolo de tal forma que se consiente un reparto homogéneo de los resultados que pretende evadir el problema a nivel de unidad. Si los mandos no tienen herramientas efectivas de gestión de recursos humanos, no se arriesgarán a ocupar el rol que el sistema les proporciona. Otra dificultar a destacar es la aplicación de la evaluación y su gestión concreta, especialmente con respecto a la remuneración de la productividad y con respecto a determinar ciertos mecanismos de progresión en la carrera profesional, a menudo no tienen el suficiente valor para incidir de forma clara en la motivación de los evaluados, quedándose en un nivel simbólico que tiende a rebajarse a partir de distribuciones más o menos lineales.

La diversidad de diseños y opciones para concretar la estrategia y los instrumentos de la evaluación debe englobar tanto a quién se va a evaluar, en base a qué, cómo y cuándo, por lo que resulta fundamental adecuar el planteamiento y objetivos a la realidad organizativa de las Administraciones Públicas, porque no es lo mismo evaluar en una administración local que en una autonómica o en la del Estado.

Javier Salinas (en prensa) hace referencia a la evaluación del rendimiento como un sistema indicador, éste señala los principales beneficios y costes que se producen tras 
la implantación de la evaluación del desempeño, y se pueden calcular en el impacto que tiene sobre el bienestar social. En cuanto a los beneficios, se espera una mayor eficacia, eficiencia y equidad del sector público. En los costes, se hace mención a los directos que son los derivados de la recogida y difusión de datos, y por otro lado los indirectos, es decir, aquellas consecuencias disfuncionales e imprevistas.

A continuación se señalan algunas de las ventajas derivadas de la implantación de un sistema de indicadores como es la evaluación del desempeño en las Administraciones Públicas:

- Esclarecer los objetivos de la organización.

- Conseguir un conocimiento superior del proceso de producción. Un análisis minucioso de los datos de rendimiento facilitaría la identificación de las modalidades de provisión y organización de servicios con las que se podría lograr un efecto más satisfactorio.

- Proporcionar la igualación del rendimiento conseguido en las diferentes organizaciones. El contar con datos referentes al rendimiento es la primera fase de la actividad de establecimiento de parámetros que permitan realizar comparaciones entre distintas unidades, lo que contribuye a que los servicios públicos aprendan de las mejores prácticas observadas.

- Iniciar la responsabilidad y transparencia de la organización ante las personas a las que facilitan sus servicios.

Por lo tanto, el objetivo de la evaluación en el sector público es causar efecto en la promoción en la carrera, la provisión y el sostenimiento de los puestos de trabajo y para la determinación de una parte de las retribuciones complementarias, que habrán de estar ligadas a la productividad o al rendimiento. En este sentido, la resultante final será siempre una dimensión de ejecución en el trabajo, que indicará el grado de realización de las conductas y el logro de objetivos asignados al empleado. Este objetivo último no será logrado si el método de evaluación no posee las características psicométricas adecuadas.

En síntesis, de esta manera, se implanta en el ordenamiento español un elemento de motivación personal y de control interno que hasta ahora le era ajeno y que es frecuente a las reformas que se están pronunciando en el resto de Europa. 


\section{$U L L L$

\section{4. ¿EXISTE RELACIÓN ENTRE LA EVALUACIÓN DEL DESEMPEÑO Y LA MEJORA DE}

LA MOTIVACIÓN DE LOS EMPLEADOS?

Para Gibson, Ivancevich, Donnelly y Konopaske (2006), la motivación es un concepto que describe las fuerzas que actúan en un individuo que le permiten iniciar y dirigir su comportamiento. Son las organizaciones las que evalúan la cooperación de sus miembros, estableciendo mecanismos que permitan disponer de una fuerza de trabajo suficientemente motivada para un desempeño eficiente y eficaz, que conduzca al logro de los objetivos y las metas de la organización y al mismo tiempo se logre satisfacer las expectativas y aspiraciones de sus integrantes (Urdaneta y Urdaneta, 2013).

Un sistema de compensación está orientado a premiar a los empleados que consigan buenos resultados para la propia organización, siendo contingente al desempeño y equitativo en dar las recompensas, por lo tanto es normal que las decisiones de compensación sean el fruto de la evaluación del desempeño. Algunos autores consideran positivo ligar la evaluación a la retribución, pero sólo para aquellos empleados de alto desempeño (Ángel, Dubin, Salvador y Simón, 2008).

Amorós (2007; citado en Urdaneta y Urdaneta, 2013) expone que la evaluación del desempeño permite a los trabajadores potenciar la motivación cuando se percibe una valoración positiva y favorable para el empleado, siendo éste uno de los indicadores que requiere mayor atención dentro de la organización. También dice que la motivación del personal en la evaluación del desempeño surte efecto cuando se aplica un sistema adecuado que permita a las personas ser conscientes del esfuerzo que emiten en la realización de las tareas, lo que resulta en un desempeño óptimo de su cargo, y cuando este desempeño a la vez es recompensado y retribuido por la organización.

La teoría de la Expectativa de Vroom explica la relación entre motivación y rendimiento. Desde esta teoría, existen dos componentes vinculados a la motivación, el comportamiento y el nivel de esfuerzo que tiene cada empleado. El individuo selecciona el nivel de esfuerzo y el comportamiento en el trabajo según las expectativas o las consecuencias positivas que espera obtener (promoción, reconocimiento, mejora salarial, etc), por lo que éste debe conocer el resultado de su trabajo para así motivarse (Dolan, Cabrera, Jackson y Schuler, 2007). 


\section{ULL}

Si a los trabajadores se les evalúa continuamente, éstos tendrán mejor disposición de saber cuáles son los conocimientos y las habilidades laborales que necesitan para disminuir los errores en el trabajo y por ende existirá una mayor motivación laboral. Por esta razón, la implantación de un sistema de evaluación del desempeño ayuda a mejorar el rendimiento, motivando a aquellos que cumplan con sus tareas.

Hellriegel y Slocum (1999) señalan que el desempeño es la consecuencia de la capacidad que posea un trabajador y aquello que le motiva. Para Robbins (1999), el éxito en el trabajo se puede ver facilitado o perjudicado por la existencia o ausencia de los recursos o materiales para trabajar. El desempeño de un empleado está en función de la interacción de la habilidad y la motivación.

Cuando existe alta motivación es porque su capacidad es el reflejo de lo que puede hacer o hará, ya que posee las herramientas suficientes para realizar cualquier actividad o tarea. En cambio, cuando no se dan estos factores se dice que existe una baja motivación, por lo que se debe capacitar a los empleados con los recursos y herramientas necesarias para que esto no suceda.

Por lo tanto, la evaluación del desempeño incluye en sí misma la valoración de las competencias, junto a las condiciones laborales y personales del trabajador a la hora de desempeñar su puesto de trabajo.

El empleado busca el reconocimiento dentro de la organización y la satisfacción de sus necesidades, al satisfacer estos dos objetivos, su motivación se convertirá en el impulsor para asumir responsabilidades y encaminar su conducta laboral a lograr metas que permitirán a la organización a lograr su razón de ser, con altos niveles de eficacia. Las motivaciones se dan cuando los objetivos de la organización y los objetivos individuales están alineados y se satisfacen mutuamente. El desarrollo de un clima organizacional que origine una motivación sostenida hacia las metas de la organización es de suma importancia, por lo que se deben combinar los incentivos propuestos por la organización con las necesidades humanas y la obtención de las metas y objetivos.

La mejora del desempeño es posible con la implantación de sistemas de remuneración justos que estén basados en los méritos, es un error suponer que a todos los trabajadores les interesan las mismas recompensas, por lo que cada vez son más las empresas que instauran planes de prestaciones a la carta: sistemas de incentivos en los 


\section{ULI

que los empleados eligen sus prestaciones de un menú de posibles opciones, esto puede ser una estrategia efectiva de motivación.

\section{CONCLUSIONES}

La evaluación del desempeño es un proceso a través del cual la organización evalúa la calidad del trabajo de sus empleados e intenta mejorar su rendimiento, actuando como un indicador del ajuste entre individuo y organización. Los objetivos son múltiples, pero la toma de decisiones, el desarrollo del empleado y el establecer metas, son objetivos fundamentales en el sistema de evaluación. Para que las empresas alcancen mayores niveles de capacidad de adaptación, flexibilidad y especialización, tienen que contar con empleados comprometidos y especializados que formen parte de equipos de trabajo, que tengan capacidad para asumir nuevos retos y ser más flexibles y tolerantes ante los cambios.

Es un instrumento de gran importancia en manos de los Recursos Humanos, los gerentes deben interesarse por la implantación y seguimiento de los múltiples aspectos del sistema de evaluación. Este método permite centrar la atención en la relación entre superior y subordinado, las facetas del trabajo y las condiciones de la organización.

Cuando se habla de dimensiones en la evaluación del desempeño se hace referencia a tres tipos, en primer lugar se encuentra el desempeño de tarea, que tiene como principal predictor la aptitud cognitiva formada por los conocimientos del puesto y la habilidad para la resolución de problemas. En segundo lugar, se encuentra el desempeño contextual, que es aquel comportamiento de naturaleza social, que actúa en el desempeño de tarea facilitándolo o dificultándolo. En tercer lugar están aquellas conductas que van en contra de los intereses legítimos de la organización, que reciben el nombre de contraproductivas.

La evaluación del desempeño cuenta con varios métodos para llevar a cabo la evaluación, según las características y los recursos económicos que posea la organización el método escogido será uno u otro. Algunos de los métodos se han quedado obsoletos por no adaptarse a las nuevas estructuras organizacionales y se han dejado de utilizar con el tiempo. Por otro lado, la entrevista es un instrumento 


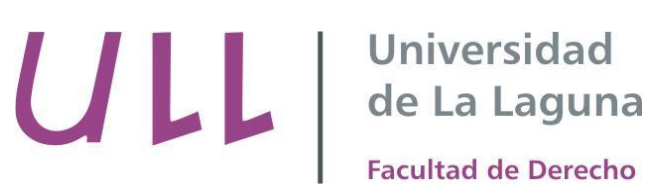

fundamental que tiene como finalidad el obtener información del evaluado, así como compartir los resultados del proceso de evaluación.

La existencia de errores en la evaluación del desempeño se puede deber por diversos motivos, uno de ellos es la falta de clarificación en los criterios. Estos errores se dan cuando el evaluador no posee la suficiente experiencia y formación, produciendo falsos resultados y afectando al proceso de evaluación.

Para que la implantación de la evaluación del desempeño sea totalmente efectiva es necesario que los empleados tengan una percepción de los objetivos, de las funciones de la evaluación, y que cada individuo se crea útil y necesario para la organización. La implicación debe ser global en toda la organización, desde la alta dirección hasta los subordinados, siendo de gran importancia la revisión continua de la calidad del sistema implantado.

La implantación de la evaluación del desempeño en las Administraciones Públicas es un reto difícil, ya que es algo nuevo y que hasta el momento parecía carecer de importancia entre los empleados públicos. Tras ser recogida en el Estatuto Básico del Empleado Público, cuenta con apoyo legal y es necesaria su implantación en las administraciones para un mejor funcionamiento. De esta manera, la evaluación del desempeño en las Administraciones Públicas aportará información del rendimiento que los empleados desarrollan y si están desempeñando de manera efectiva sus tareas.

La relación que existe entre motivación y evaluación del desempeño está vinculada a los resultados que el empleado obtenga de la evaluación, los incentivos, las compensaciones, etc. Partiendo de la base de que cada empleado se siente motivado o desmotivado por factores diferentes, por lo general el incentivo qué más motiva es el dinero (aumento de salario, promoción, bonos, etc.), por ejemplo: si el empleado que desempeña su tarea de forma óptima y en consecuencia obtiene resultados en la evaluación positivos, éste es premiado con un incentivo de aumento salarial y por consiguiente estará motivado por realizar muy bien su tarea en la organización. De otra manera, cuando los resultados de la calificación son negativos se debe dar una retroalimentación al empleado para que pueda modificar los fallos y de esta manera mejore en el futuro, si la retroalimentación no se hace o se hace de forma equívoca puede producir desmotivación en el empleado. La información sobre cómo se está llevando a cabo el trabajo es motivadora en sí misma, ya que permite continuar en esa 
dirección, o bien replantear la forma de hacer las cosas, teniendo claro en qué se puede mejorar.

La evaluación del desempeño es un instrumento que deber ser valorado por la organización (directivos y empleados) y por los recursos humanos, ya que la implantación es necesaria para conocer el rendimiento y el desempeño de los empleados, sus potenciales y sus deficiencias. El mercado de trabajo está pasando un mal momento debido a la crisis económica que sufre el país, es por ello que los empleados adoptan una actitud negativa frente a la evaluación del desempeño, pero son las empresas las que deben contar con las herramientas y los recursos económicos suficientes para llevar a cabo este sistema y lograr que los empleados lo consideren positivo.

La información obtenida de la evaluación debe ser utilizada de manera efectiva para la toma de decisiones y para la retroalimentación que se debe hacer a los empleados para que puedan mejorar aquello en lo que son deficientes y para que puedan ser recompensados si la empresa así lo estipula, siendo los incentivos el aspecto más importante para los trabajadores. El principio de igualdad es uno de los más importantes para que el funcionamiento y la transparencia del sistema sea fiable y eficaz, ya que todos los empleados deben ser tratados de la misma manera sin importar raza, edad y sexo. En definitiva, cada vez son más las empresas que implantan este sistema para lograr: unos empleados que desempeñen las tareas del puesto con total eficacia, una mejor productividad, competitividad con otras empresas, información que será utilizada para diversos fines como la reconstrucción de los puestos. Por lo que la evaluación del desempeño es una ventaja que proporcionará una visión hacia al futuro, obteniendo tanta importancia que está reflejada en el Estatuto del Empleado Público para ser implantada en las Administraciones Públicas y así lograr una mayor eficacia en los empleados públicos. 


\section{ULL}

\section{REFERENCIAS BIBLIOGRÁFICAS}

- Ángel, A., Dubin, K., Salvador, M. y Simón, C. (2008). La evaluación del desempeño en las Administraciones Públicas. Madrid: Centro PwC \& IE del Sector Público.

- Borman, W.C., y Motowidlo, S.J. (1993). Expanding the criterion domain to include elements of contextual performance. En N. Schmitt, y W.C. Borman (Eds.), Personnel selection in organizations (71-98). San Francisco, CA: Jossey-Bass.

- Borman, W.C., Penner, L.A., Allen, T.D. y Motowidlo, S.J. (2001). Personality predictors of citizenship performance. International Journal of Selection and Assessment, Vol. 9, 52-69.

- Campbell, J.P. y Kuncel, N.R. (2001). Individual and team training. En N.Anderson, D. S. Ones, H. K. Sinangil y C. Viswesvaran, (Eds), Handbook of Industrial, Work and Organizational Psychology (pp. 278-312). London: SAGE.

- Carroll, S.J. y Schneier, C.E. (1982). Performance appraisal and review systems: The identification, measurement and development of performance in organizations. Scott, Foresman Series in management and organizations.

- Chiavenato, I. (2007). Administración de recursos humanos. El capital humano de las organizaciones (octava edición). México: Mc Graw Hill

- Cleveland, J.N., Murphy, K.R., y Williams, R.E. (1989). Multiple uses of performance appraisal: Prevalence and correlates. Journal of Applied Psychology, 74, 130-135.

- Dolan, S. L., Cabrera, R. V., Jackson, S. E., y Schuler, R. S. (2007). La Gestión de los Recursos Humanos. Cómo atraer, retener y desarrollar con éxito el capital humano en tiempos de transformación (tercera Edición). Editorial Mc Graw Hill, Madrid.

- Gibson, J; Ivancevich, J.; Donnelly, J.; y Konopaske, R. (2006). Organizaciones. Comportamientos, estructura, procesos (duodécima edición). México: Editorial. Mc Graw Hill.

- Gorriti, M. (2007). La Evaluación del Desempeño en las Administraciones Públicas Españolas. Revista de Psicología del Trabajo y de las Organizaciones, 23(3), 367-387.

- Hellriegel, D., Slocum, J. y Woodman, R. (1999). Comportamiento organizacional (octava edición). México: Thomson. 


\section{UIII Uninesisiad

- Jones, G. y George, J. (2006). Administración Contemporánea (cuarta edición), México: Mc Graw Hill.

- Murphy, K. R. (1990). Job performance and productivity. En K. R. Murphy y F.

E. Saal (Eds). Psychology in Organizations: Integrating science and practice. (pp. 157176). Hillsadle, N.J.: Erlbaum.

- Ones, D.S., Viswesvaran, C. y Dilchert, S. (2005). Cognitive ability in personnel selection decisions. En A. Evers, N. Anderson y O. Voskuijl (Editores), The Blackwell Handbook of Personnel Selection. (pp. 143-173). London: Blackwell.

- Robbins, S. P. (1999). Comportamiento Organizacional (octava edición). México: Prentice-Hall.

- Sackett, P.R. y DeVore, C. J. (2001).Counterproductive behaviors at work. En N. Anderson, D. S. Ones, H. K. Sinangil y C. Viswesvaran (Ed.). Handbook of Industrial, Work and Organizational Psychology (pp. 145-164). London: SAGE

- Salgado, J. F., y Cabal, A. L. (2011). Evaluación del Desempeño en la administración Pública del Principado de Asturias: Análisis de las Propiedad Psicométricas. Revista de Psicología del Trabajo y de las Organizaciones, 27, 75-91.

- Salinas, J. (en prensa). El empleo público en España: evaluación del desempeño, incentivos y retribuciones. Manuscrito no publicado.

- Schmidt, F.L. y Hunter, J.E. (1998). The validity and utility of selection methods in personnel psychology: Practical and theoretical implications of 85 years of research findings. Psychological Bulletin, 124 (2), 262-274.

- Society for Industrial and Organizational Psychology, Inc. (1987). Principles for the validation and use of the personnel selection procedures (tercera edición). College Park, MD.

- Steensma, H, y Otto, L. (2000). Perception of Performance Appraisal by Employees and Supervisors: Self-Serving Bias and Procedural Justice. Journal of Collective Negotiations, vol. 29 (4), 307-319.

- Urdaneta, O. R. y Urdaneta, M.V. (2013). Evaluación del desempeño y motivación del personal en los Institutos de Investigaciones de Salud. Revista de Ciencias Sociales, Vol. XIX (4), 672-682. 